\title{
Throughput Optimization for Grant-Free Multiple Access with Multiagent Deep Reinforcement Learning
}

\author{
Vincent Wong \\ Professor, Electrical and Computer Engineering \\ The University of British Columbia \\ Vancouver, BC, Canada, V6T 1Z4
}

\begin{abstract}
Grant-free multiple access (GFMA) is a promising paradigm to efficiently support uplink access of Internet of Things (IoT) devices. In this talk, we present a deep reinforcement learning (DRL)-based pilot sequence selection scheme for GFMA systems to mitigate potential pilot sequence collisions. We formulate a pilot sequence selection problem for aggregate throughput maximization in GFMA systems with specific throughput constraints as a Markov decision process (MDP). By exploiting multiagent DRL, we train deep neural networks (DNNs) to learn near-optimal pilot sequence selection policies from the transition history of the underlying MDP without requiring information exchange between the users. While the training process takes advantage of global information, we leverage the technique of factorization to ensure that the policies learned by the DNNs can be executed in a distributed manner. Simulation results show that the proposed scheme can achieve an average aggregate throughput that is close to the optimum, and has a better performance than several heuristic algorithms.
\end{abstract}

